\title{
Mouse intestinal microbiota reduction favors local intestinal immunity triggered by antigens displayed in Bacillus subtilis biofilm
}

\author{
Cédric M. Vogt ${ }^{1}$, Monika Hilbe ${ }^{2}$, Mathias Ackermann ${ }^{1}$, Claudio Aguilar ${ }^{3}$ and Catherine Eichwald ${ }^{1 *}$ (i)
}

\begin{abstract}
Background: We previously engineered Bacillus subtilis to express an antigen of interest fused to TasA in a biofilm. B. subtilis has several properties such as sporulation, biofilm formation and probiotic ability that were used for the oral application of recombinant spores harboring Echinococcus granulosus paramyosin and tropomyosin immunogenic peptides that resulted in the elicitation of a specific humoral immune response in a dog model.

Results: In order to advance our understanding of the research in oral immunization practices using recombinant B. subtilis spores, we describe here an affordable animal model. In this study, we show clear evidence indicating that a niche is required for $B$. subtilis recombinant spores to colonize the densely populated mice intestinal microbiota. The reduction of intestinal microbiota with an antibiotic treatment resulted in a positive elicitation of local humoral immune response in BALB/C mice after oral application of recombinant B. subtilis spores harboring TasA fused to $E$. granulosus (102-207) EgTrp immunogenic peptide. Our results were supported by a lasting prevalence of spores in mice feces up to 50 days after immunization and by the presence of specific secretory lgA, isolated from feces, against E. granulosus tropomyosin.
\end{abstract}

Conclusions: The reduction of mouse intestinal microbiota allowed the elicitation of a local humoral immune response in mice after oral application with spores of B. subtilis harboring immunogenic peptides against $E$. granulosus.

Keywords: Bacillus subtilis, TasA, Biofilm, Spores, Oral immunization, IgA, Antigen, Microbiota

\section{Background}

Bacillus subtilis is a Gram-positive bacterium that has several attractive properties with high potential in bioapplications such as vaccines [1-3] or bioremediation $[4,5]$. One of these properties is the capacity to form endospores upon nutrient starvation [6, 7]. The B. subtilis spores have been vastly adopted as a carrier in immunization strategies because of their resistance to harsh conditions as low $\mathrm{pH}$, high temperature, and noxious chemicals. In this context, methods as decoration of the spores by direct fusion with different coat proteins $[8,9]$

\footnotetext{
*Correspondence: ceichwald@vetvir.uzh.ch

1 Institute of Virology, University of Zurich, Winterthurerstrasse 266a, 8057 Zurich, Switzerland

Full list of author information is available at the end of the article
}

or adsorption [10-12] with the antigen of interest illustrate the versatility of the spores as an antigen carrier. Another property of $B$. subtilis is the ability to form architecturally complex communities termed biofilms, which self-produce an extracellular matrix comprised of lipids, proteins exhibiting amyloid-like properties, extracellular DNA and exopolysaccharides [13]. Interestingly, evidence suggests that $B$. subtilis can develop biofilms in the gut of living organisms $[14,15]$. This is true for some nondomesticated laboratory B. subtilis strains as NCIB 3610 $[16,17]$. We recently showed that it is possible to express heterologous proteins in a B. subtilis biofilm by fusion to the C-terminus of the biofilm matrix protein, TasA [18]. Optimized expression of the heterologous protein was established using a $\operatorname{tas} A / \sin R$ genetic background, where Sin $R$ is a repressor of the tap $A$-sip $W$-tas $A$ operon, among 
other genes $[17,19]$. A third feature corresponds to its probiotic properties in human and livestock [20-22]. By combining these properties, we recently showed that it is possible, in a dog model, to elicit a local humoral immune response against enteric antigens such as E. granulosus parasite [23]. In this dog model, the recombinant spores were able to bypass the stomach barrier and then form, after germination, a biofilm in the intestine that displayed an antigen within its matrix, allowing the stimulation of gut-associated lymphoid tissue (GALT) and thereby, eliciting a local humoral immune response.

In the present study, we tested the local humoral immune response in the intestine of $\mathrm{BALB} / \mathrm{c}$ mice, orally applied with recombinant spores of $B$. subtilis harboring a TasA fusion to the E. granulosus immunogenic peptide (102-207) EgTrp. We show that the elicited intestinal humoral immune response is favored when providing a niche to recombinant spores in the intestinal microbiota.

\section{Results}

Oral application of recombinant $B$. subtilis spores in mice We first showed that B. subtilis was found in the mice intestine expressing TasA, even after the oral inoculation with recombinant $B$. subtilis tasA/lux/TasAmCherry ([23] and Table 1) and that recombinant vegetative cells were not found in mice feces after 6 days post-inoculation (Additional file 1: Figure S1a and b). Our data also show that $64-93.5 \%$ of the orally applied recombinant $B$. subtilis spores are retained in the mice gut (Additional file 1: Figure S1c and d). Additionally, no pathologic changes, like inflammatory, degenerative or neoplastic changes were observed in the small or in the large intestine sections of all mice treated with recombinant $B$. subtilis spores when compared with untreated animals (Additional file 1: Figure S1e). Collectively, our data suggest that spores germinate in the gut of mice. To investigate if recombinant spores of $B$. subtilis tasA/sinR (102-207) EgTrp, hereafter named $B$. subtilis (102-207)EgTrp, (Fig. 1a and Table 1) can elicit a local intestinal humoral immune response in mice, we provided the recombinant spores via oral gavage. For this purpose and as depicted in Fig. 1b, three groups of mice were treated at days 1,21 and 42 with saline solution (placebo), or with $5 \times 10^{10} \mathrm{CFU}$ of recombinant spores of $B$. subtilis tas $A / \sin R$ (group tasA/sinR) or $5 \times 10^{10} \mathrm{CFU}$ of recombinant spores of $B$. subtilis (102-207)EgTrp (group (102-207)EgTrp). Mice were isolated into individual cages to determine the number of shed spores after oral application and feces were collected every $24 \mathrm{~h}$ until day 6 and also on days 20, 41 and 50 of the scheduled treatment. As observed in Fig. 1c, mice applied with recombinant spores of $B$. subtilis tas A/sinR or (102-207)EgTrp exhibited a decline in the shedding of recombinant spores after 4 days post-application. Additionally, we estimated that the total number of recombinant spores of B. subtilis tasA/sinR or (102-207) EgTrp strains retained in the gut correspond to $91 \%$ and $99 \%$, respectively. We could not detect recombinant spores in the feces of animals before the subsequent oral applications (days 21 and 42) or at experiment termination (day 50) (Fig. 1d). Next, we tested if mice could elicit a humoral intestinal immune response against E. granulosus antigen EgTrp by testing immunoglobulins extracted from feces at day 50 through indirect ELISA coated with B. subtilis biofilm extracts expressing (102-207)EgTrp antigen, biofilm extract of B. subtilis tasA/sinR, recombinant $\mathrm{H}_{6}$-EgTrp peptide or an irrelevant antigen such is $\mathrm{H}_{6}$-mCherry. None of the experimental animal groups showed a positive elicitation of the local humoral immune response against the tested antigens (Fig. 1e). Similar results were obtained when tested the mice sera of day 50 , which unveil no elicitation of the humoral immune response of neither IgG (Additional file 2: Figure S2a) nor IgA (Fig. 1f). Also, the animals remained healthy during the whole procedure as denoted by the lack of difference in the mean body weight among the experimental groups (Additional file 2: Figure S2c) and the absence of symptoms such as diarrhea, hypothermia, and mastocytosis. Thus, the recombinant spores of B. subtilis seem not able to elicit an immune response under the conditions tested in these experiments.

\section{Table 1 Bacillus subtilis strains used in this study}

\begin{tabular}{|c|c|c|}
\hline Strain & Genotype $^{a}$ & Reference/ source \\
\hline $\operatorname{tas} A / \sin R$ & $\operatorname{tas} A-\sin R:: K^{r}$ & Vogt et al. [18] \\
\hline tasA/sinR/TasA-(102-207)EgTrp & tasA-sinR::Kmr; amyE::yqxM-sipM-tasA-(102-207)EgTrp Spc ${ }^{r}$ & Vogt et al. [18] \\
\hline wt/lux & lacA::P $P_{\text {tasA }}-l u x C D A B E E r m^{r}$ & Vogt et al. [23] \\
\hline tasA/lux/TasA-mCherry & 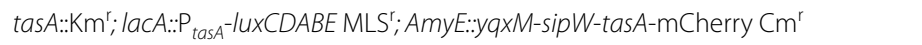 & Vogt et al. [23] \\
\hline $\operatorname{tas} A / \sin R / l u x / T a s A-m C h e r r y$ & 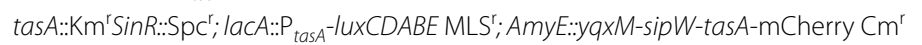 & Vogt et al. [23] \\
\hline
\end{tabular}

a $\mathrm{Km}^{\mathrm{r}}$ : kanamycin resistance; $\mathrm{Spc}$ : spectinomycin resistance; $\mathrm{Cm}^{\mathrm{r}}$ : chloramphenicol resistance; $\mathrm{MLS}^{\mathrm{r}}$ : macrolide-lincosamide-streptogramin B (erythromycin and lincomycin) resistance 


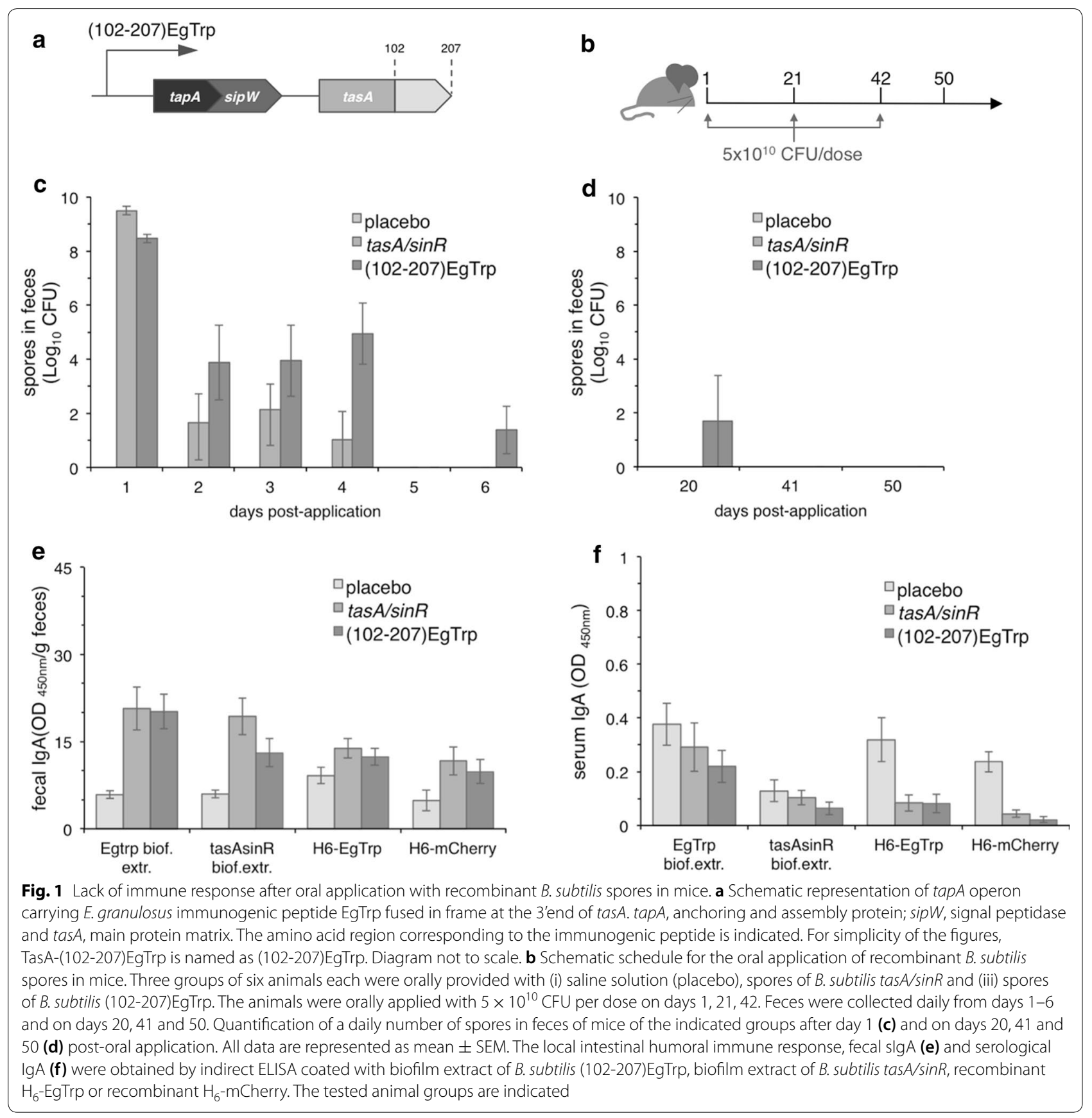

Reduction of the intestinal microbiota favors local immune response after oral application of recombinant $B$. subtilis spores in mice

To stimulate the GALT with a consequent elicitation of a humoral immune response against the presented antigens, the spores of $B$. subtilis require a niche where to germinate, colonize and form a biofilm. However, the efficiency of this process may be severely hampered by the densely populated host intestinal microbiota, which could be actively competing for the same niche. We hypothesized that this competition could be of different nature such as for nutrient availability [24] or space competition, impeding the colonization of new bacterial species [25]. In any of these circumstances, the reduction of the intestinal microbiota before the oral application of recombinant spores of $B$. subtilis could provide a chance for successful colonization and subsequent biofilm formation, which in turn may elicit a local humoral immune 
response. To test this hypothesis, we treated mice for five consecutive days with an antibiotic cocktail [26]. The effective reduction of the intestinal microbiota was corroborated by the absence of bacterial colonies isolated from feces in three different culture media such as LuriaBertani, brain heart infusion and nutrient broth (Additional file 3: Figure S3 a, b and c). Of note, the antibiotic treatment had no detrimental effect on the mice health as denoted by similar bodyweight among untreated and treated animals (Additional file 3: Figure S3d). Thus, as described in Fig. 2a, all groups of mice were first treated for 5 days with an antibiotic cocktail, followed by three oral applications of $5 \times 10^{10} \mathrm{CFU}$ of recombinant spores per dose on days 1,21 and 42. All animals were sacrificed on day 50 . The animals were divided, as above, into three experimental groups: (i) placebo, (ii) $\operatorname{tas} A / \sin R$ and (iii) (102-207)EgTrp. As in the previous experiment, mice shed recombinant spores until the fourth day from the

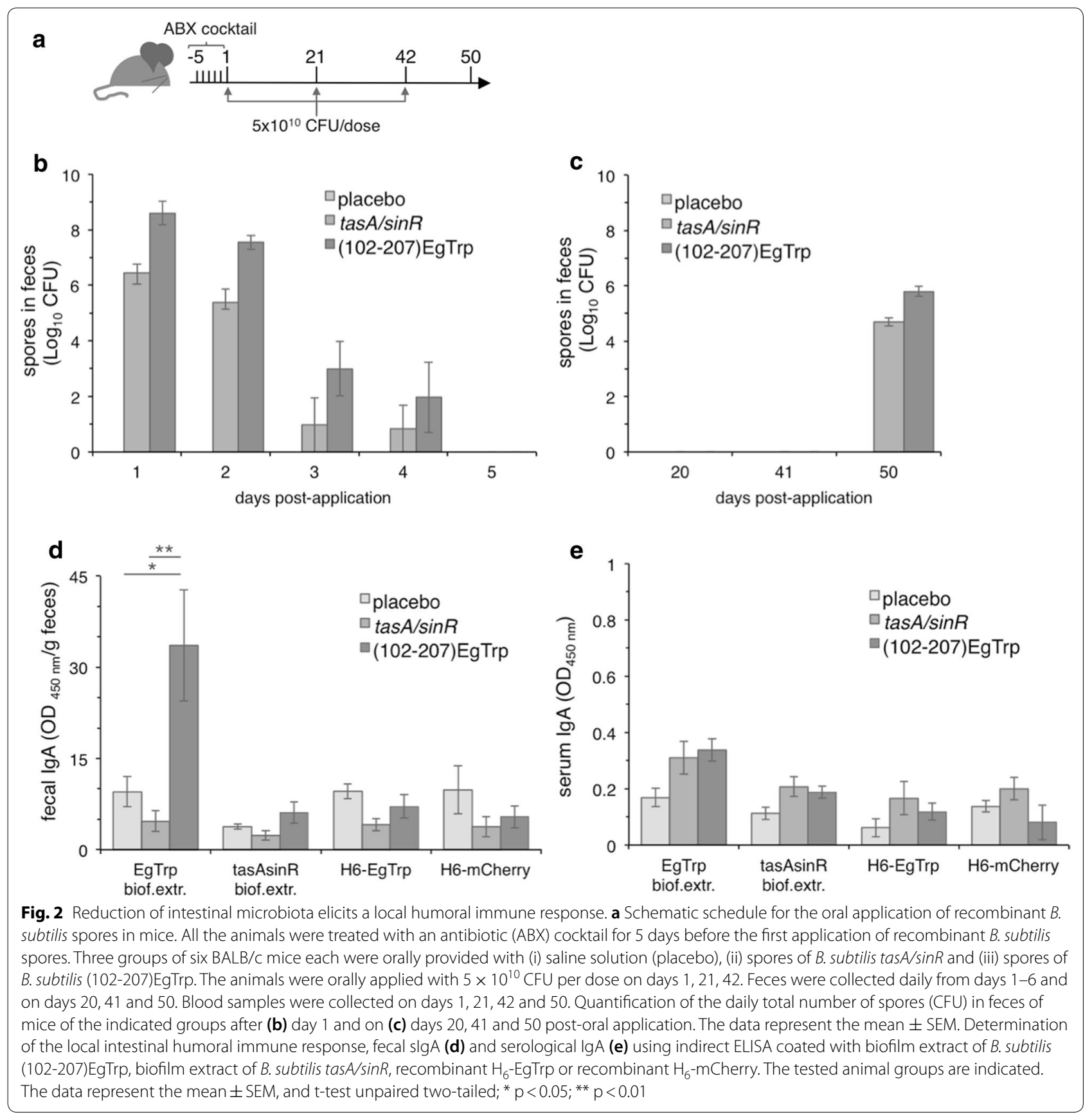


first oral application (Fig. 2b). The number of recombinant spores retained in the gut was $99.9 \%$ for B. subtilis $\operatorname{tas} A / \sin R$ and $97.6 \%$ for (102-207)EgTrp. Surprisingly, recombinant spores from both tasA/sinR and (102-207) EgTrp groups were readily detected at day 50 in the mice feces (Fig. 2c). The local intestinal humoral immunity was determined using an indirect ELISA coated with either an EgTrp biofilm extract, tas $A / \sin R$ biofilm extract, recombinant $\mathrm{H}_{6}$-EgTrp or recombinant $\mathrm{H}_{6}$-mCherry. Interestingly, the secretory $\operatorname{IgA}(\operatorname{sg} \mathrm{A})$ isolated from feces of mice of the (102-207)EgTrp group recognized specifically the biofilm extracts harboring the heterologous expression of the immunogenic peptide EgTrp (Fig. 2d). In the same conditions, no recognition by sIgA isolated from mice feces of the placebo and $\operatorname{tas} A / \sin R$ groups was observed for EgTrp expressed in biofilm extracts. Importantly, the lack of TasA and SinR in biofilm extracts (i.e., tas $A / \sin R$ biofilm extracts), resulted in no local humoral response for the three tested groups. Additionally, no response for sIgA was obtained for $\mathrm{H}_{6}$-EgTrp or $\mathrm{H}_{6}$-mCherry. Mice did not induce any serological humoral immune response for the tested antigens in an IgG (Additional file 1: Figure S1b) or IgA context (Fig. 2e). Between the experimental groups, no differences were observed in animal body weight (Additional file 2: Figure S2d), as well as no signs of diarrhea, hypothermia or mastocytosis after pretreatment with antibiotics, followed by oral application of recombinant $B$. subtilis spores.

\section{Discussion}

It is of high interest to perform antigen tests aimed at vaccine development in affordable animal models such as rodents, being mice (as BALB/c strain) frequently the model of choice. Initial evidence suggested that recombinant B. subtilis spores germinate in the gut of BALB/ c mice without generating adverse effects. Therefore in an attempt to evaluate the effectiveness of our method, based on the display of antigens in biofilms, we used a previously tested recombinant $B$. subtilis (102207)EgTrp that elicited a positive humoral immune response in dogs [23]. The antigen (102-207) EgTrp corresponds to tropomyosin, an immunogenic peptide of Echinococcus granulosus, described previously by Pétavy and collaborators $[27,28]$. E. granulosus cestode is the causative agent for cystic echinococcosis, responsible for high human morbidity and mortality and having a high economic impact on livestock [29-31]. In our first trial in mice, we could not detect an elicitation of the local immune response after three oral doses of recombinant $B$. subtilis spores. This observation was not surprising, and it is consistent with previous observations [9, 32-39], where even nine consecutive oral doses with B. subtilis spores evoked weak or null immune response compared to similar conditions after intranasal immunization in mice [8]. The pre-treatment with antibiotics to reduce the density of gut microbiota is a standard procedure in other mouse models of enteric infection $[9,40]$. In our experimental setting, we administrated an antibiotic cocktail [26] for 5 days before the first oral application of recombinant $B$. subtilis spores. The persistence of the recombinant spores in the gut was determined by their prevalence in feces at day 50 post-application compared with the mice untreated with antibiotics in the same conditions. The spore persistence suggests that recombinant $B$. subtilis spores were able to find a niche in the gut (colonization), followed by germination, biofilm formation, and subsequent sporulation. Additionally, an elicitation of local humoral immune response as determined by the presence of specific sIgA recognizing EgTrp. Therefore, the decrease of the intestinal microbiota of mice, colonized for at least 1000 different bacterial species (40), is a requisite for the settlement of recombinant B. subtilis spores. Previous evidence demonstrates that B. subtilis naturally colonize the gut of humans [14], dogs [23], and even grass carps [41], suggesting that B. subtilis cannot be considered as an allochthonous microorganism. Interestingly, the generated immunoglobulins recognized the EgTrp antigen peptides only when present in a biofilm extract but not the one from the recombinant purified $\mathrm{H}_{6}-(102-278)$ EgTrp. In this context, we recently showed [23] that dogs treated orally with recombinant spores of B. subtilis (102-207) Egtrp elicited a humoral immune response recognizing specifically recombinant purified $\mathrm{H}_{6}$-EgTrp. Our results suggest that the biofilm TasA-(102-207)EgTrp fusion displayed in the gut mice got selected by immunoglobulins through specific conformation that is not forged by $\mathrm{H}_{6}$-EgTrp $[42,43]$. This outcome also confirms the dissimilarities between the intestinal immune system of mice and that of dogs. It is important to highlight that the results obtained here cannot necessarily apply to a different animal model. The specificity of the humoral response was denoted by the lack of recognition of unrelated antigens as $\mathrm{H}_{6}$-mCherry by sIgA. It is of note that we could not distinguish a serum-specific IgA in any of the experimental settings tested. As expected, the use of recombinant $B$. subtilis spores resulted safe in mice. Our recombinant spores under the conditions described do not alter the health status of treated mice when compared to its control pairs, as denoted by no discrepancy in their body weight, pathological states (like hypothermia, diarrhea or mastocytosis) and by infiltration of inflammatory cells in the small and large intestine. 


\section{Conclusions}

In this work, we provide evidence that a reduction in the intestinal microbiota in mice could settle the basis for favoring an oral immunization using recombinant $B$. subtilis spores.

\section{Methods}

\section{Ethics statement}

All the mouse experiments were performed according to the guidelines of the animal experimentation law (SR 455.163; TVV) of the Swiss federal government. The Cantonal Veterinary Office of Zurich, Switzerland, approved the protocols under animal experimentation number $104 / 2012$.

\section{Bacillus subtilis strains, media and culture conditions}

The B. subtilis strains used in this study were previously described by Vogt et al. [18]. For routine growth and spore quantification, cells were propagated on Luria-Bertani (LB) medium. The final concentrations of antibiotics used for the B. subtilis strains were as follows: $100 \mu \mathrm{g} /$ $\mathrm{ml}$ for spectinomycin (Spc) and $10 \mu \mathrm{g} / \mathrm{ml}$ for kanamycin $(\mathrm{Km})$.

\section{Plasmid constructions}

pQE32-(102-278)EgTrp and pQE32-mCherry were previously described by Vogt et al. [23].

\section{Production of recombinant $B$. subtilis spores}

The recombinant spores were produced and purified as described by Vogt et al. [18].

\section{Experimental administration of recombinant $B$. subtilis spores in mice}

Intragastric gavage was orally applied to groups of six mice (females, BALB/c, 6 weeks old) with $5 \times 10^{10} \mathrm{CFU}$ of recombinant spores of $B$. subtilis in a final volume of $200 \mu \mathrm{l}$ PBS per dose on days 1, 21 and 42. The placebo group was orally applied with $200 \mu \mathrm{l}$ PBS. Mice were isolated into single cages for collection of feces every $24 \mathrm{~h}$, on days 1 to 6 and on days 20,41 and 50. Blood samples were collected by tail-bleeding the day before the application of a spores dose and at day 50. The serum was used to test the humoral response against the antigen of interest. All animals were sacrificed on day 50 post-application.

\section{Mouse intestinal microbiota reduction}

When indicated, mice were treated with an antibiotic mixture for the elimination of the gut microbiota as described by Shan et al. [26]. For this purpose, a mixture containing $0.5 \mathrm{mg} / \mathrm{ml}$ ampicillin (PanReac AppliChem, Spain), $0.5 \mathrm{mg} / \mathrm{ml}$ gentamycin (PanReac AppliChem,
Spain), $0.25 \mathrm{mg} / \mathrm{ml}$ vancomycin (Alfa Aesar, Germany), metronidazole (Alfa Aesar, Germany), and $20 \mathrm{mg} / \mathrm{ml}$ sucrose (Sigma) dissolved in sterile drinking water was provided as source of water to mice for 5 days. The reduction of the intestinal microbiota was monitored by counting the number of bacterial colonies isolated from feces in non-selective media, such as LB, heart-brain infusion and nutrient broth semi-solid media.

\section{Quantification of spores in feces}

Feces of single-caged mice were collected throughout $24 \mathrm{~h}$. The feces were resuspended to a concentration of $0.2 \mathrm{~g} / \mathrm{ml}$ in PBS, homogenized with vortex for $30 \mathrm{~s}$. Then, a $300 \mu \mathrm{l}$ aliquot of feces resuspension was heated for $20 \mathrm{~min}$ at $80{ }^{\circ} \mathrm{C}$ to kill vegetative cells. Serial dilutions were plated on selective semi-solid LB agar containing $10 \mu \mathrm{g} / \mathrm{ml}$ kanamycin to determine viable recombinant spores. The spore number per gram of feces was obtained dividing the colony forming units (CFU) by the amount in grams of feces.

\section{Indirect ELISA}

The assays were performed as described in detail by Vogt et al. [23]. For the extraction of secretory antibodies (sIgA), fecal samples were collected throughout $24 \mathrm{~h}$ from mice isolated in single cages and stored at $-20{ }^{\circ} \mathrm{C}$ until analysis. One gram of feces was resuspended in $5 \mathrm{ml}$ PBS, vortex homogenized for $30 \mathrm{~s}$ and centrifuged at $800 \times g$ for $10 \mathrm{~min}$. Then, $360 \mu \mathrm{l}$ of recovered supernatant was mixed with $40 \mu \mathrm{l}$ of feces buffer (1\% BSA, $0.01 \%$ Triton X-100, $0.1 \%$ 2-mercaptoethanol and protease inhibitor (cOmplete $^{\mathrm{TM}}$ EDTA-free protease inhibitor cocktail, Roche, Switzerland)) and kept in ice. Recombinant purified protein (500 ng/well) or $72 \mathrm{~h}$ biofilm extract $\left(\mathrm{OD}_{600 \mathrm{~nm}}\right.$ of $0.0002 /$ well) in $0.2 \mathrm{M}$ bicarbonate buffer $\mathrm{pH} 9.4$ was coated for $16 \mathrm{~h}$ at $4{ }^{\circ} \mathrm{C}$ in a 96 well multi-well plates (Nunc-Immuno Maxisorp, Thermo Scientific). Plates were incubated for $2 \mathrm{~h}$ at room temperature with blocking buffer (1\% BSA in PBS). A $100 \mu$ aliquot of feces mixture was incubated for $2 \mathrm{~h}$ at $37^{\circ} \mathrm{C}$. This was followed by incubation of the plates for $1 \mathrm{~h}$ at $37{ }^{\circ} \mathrm{C}$ with goat anti-mouse IgA conjugated to peroxidase (diluted 1:600, Sigma) or rabbit anti-mouse IgG (whole molecule) conjugated to peroxidase (diluted 1:2000, Sigma) both diluted in 1\% BSA-PBS. The plates were incubated with $100 \mu \mathrm{l}$ per well of 3,3,5,5'-tetramethylbenzidine (TMB) substrate (ThermoFisher Scientific) in the dark for $30 \mathrm{~min}$ at room temperature, and the reaction was stopped by the addition of $100 \mu \mathrm{l} 1 \mathrm{M} \mathrm{H}_{2} \mathrm{SO}_{4}$. The plates were read using an SLT 340 ATTC Tecan microplate reader (Tecan US Inc.) at an $\mathrm{OD}_{450 \mathrm{~nm}}$. The data were analyzed and processed using Microsoft ${ }^{\circledR}$ Excel $^{\circledR}$ for MAC 2011. The cut-off was determined as the average of three negative controls. The negative control value was obtained by incubation of 
the antigen followed by the secondary antibody conjugated to HRP. The cut-off was subtracted from all the sample values. Each value has been subtracted from its corresponding pre-immune (PI) value.

\section{Expression and purification of $\mathrm{H}_{6}$-tagged proteins}

$\mathrm{H}_{6}$-EgTrp and $\mathrm{H}_{6}$ - $\mathrm{mCherry}$ were expressed in Escherichia coli M15 (pREP4) (Qiagen) transformed with pQE32(102-278)EgTrp and pQE32-mCherry, as described in detail by Vogt et al. [23].

\section{Preparation of biofilm extract}

For the preparation of biofilms, cells were scraped from overnight growth on LB-agar plates, resuspended in LB liquid medium to an $\mathrm{OD}_{600 \mathrm{~nm}}$ of 1 , and then $2 \mu \mathrm{l}$ of this suspension was spotted on MSgg solid medium [16]. Biofilms were incubated at $30{ }^{\circ} \mathrm{C}$. At $72 \mathrm{~h}$, the biofilm was harvested in $0.2 \mathrm{M}$ bicarbonate buffer $\mathrm{pH} 9.4$ and dispersed using mild sonication conditions $(1 \mathrm{~min}$ at $14 \mathrm{kHz}$ ) to obtain a homogeneous resuspension. For the coating of ELISA plates, biofilm extracts were normalized to $\mathrm{OD}_{600 \mathrm{~nm}}$ of $2 \times 10^{-4}$.

\section{Histology and immunohistochemistry}

The mice's intestines were sectioned in duodenum, jejunum, ileum, cecum, and colon. Each intestinal section was knotted in both ends previous to sectioning to avoid the loss of the intestinal content. Samples of approximately $2 \mathrm{~cm}$ in length were fixed in $4 \%$ formaldehyde. After fixation, each sample was dehydrated in alcohol solutions of increasing concentration and embedded in paraffin. The embedded samples were thin-sectioned at $2-3 \mu \mathrm{m}$ and stained with hematoxylin and eosin.

For immunohistochemistry of the mice's intestinal section, the samples were de-paraffinized, rehydrated and incubated for $30 \mathrm{~min}$ at room temperature with the primary antibody (rabbit anti-TasA serum). A detection kit, containing the secondary antibody and aminoethyl carbazole as chromogen, was subsequently applied according to the manufacturer's protocols (Peroxidase/AEC Rabbit/Mouse Kit, DAKO). Images were acquired using an Olympus CX41 light microscope equipped with a 40X objective lens and an Olympus Vanox-S AxioCam interface. The acquired images were processed using Image $J$ software (Wayne Rasband, NIH, USA; http://imagej.nih. gov/ij).

\section{Additional files}

Additional file 1: Figure S1. Tracking of Bacillus subtilis recombinant in the gut of mice. Two mice were orally applied with $5 \times 10^{10} \mathrm{CFU}$ spores/ dose of B. subtilis wild-type (wt) or tasA lux TasA-mCherry strains. (a)
Feces were monitored for luminescence at 7 days post-application of recombinant spores. A color luminescence scale is shown at the right of the panel. The luminescent images were acquired with a Xenogen IVIS camera and analyzed using Living Image ${ }^{\circledR} 4.0$ software (Caliper Life Sciences, USA) (b) Immunohistochemistry of mice cecum sections incubated with anti-TasA sera (1:100) followed with secondary anti-rabbit-HRP and stained with diaminobenzidine (brown). The cell membranes and nuclei were counterstained with eosin (red)/hematoxylin (blue). Scale bar is 100 $\mu \mathrm{m}$. (c) Schematic representation of mice oral application of recombinant B. subtilis spores schedule. Mice (six females BALB/c, 6 weeks old per group) were treated $5 \times 10^{10} \mathrm{CFU}$ spores/ dose on days 1,21 and 42. The experimental groups were the following with (i) placebo (saline solution), (ii) wild-type, (iii) tasA lux TasA-mCherry and (iv) tasA sinR lux TasA-mCherry. On day 50, animals were sacrificed. (d) Plot of the total number of shed $B$. subtilis spores (CFU) in feces after each oral application. Data represent the mean \pm SD for each group of animals. (e) Representative histological sections stained with hematoxylin and eosin from intestinal samples of dogs orally inoculated with recombinant B. subtilis spores (placebo, wt, tasA lux TasA-mCherry and tasAsinR lux TasA-mCherry). Lm, intestinal lumen. Scale bar is $100 \mu \mathrm{m}$.

Additional file 2: Figure S2. IgG humoral response. Determination of the serological IgG response by indirect ELISA of mice untreated (a) or pretreated with $A B X s$ (b) before oral application of recombinant $B$. subtilis spores. The plates were coated with biofilm extract of $B$. subtilis strain (102-207)EgTrp, biofilm extract of B. subtilis tasA/sinR, recombinant purified $\mathrm{H}_{6}$-EgTrp or recombinant purified $\mathrm{H}_{6}$-mCherry. The tested animal groups are indicated. The body weight curve of the indicated mice groups untreated (c) or pretreated (d) with antibiotics before oral application with recombinant $B$. subtilis spores. The data represent the mean \pm SEM and t-test unpaired two-tailed.

Additional file 3: Figure S3. Antibiotic (ABX) cocktail treatment severely reduces bacterial microflora. One mouse (untreated, solid dark circle) and two mice ( $A B X 1$, solid dark square and $A B X 2$, open square) were untreated or treated with an antibiotic cocktail (ampicillin, gentamycin, vancomycin, and metronidazole) in the drinking water, respectively. Feces samples of individual cages were collected every day, diluted in PBS and cultured by serial dilution in enriched media as (a) Luria-Bertani, (b) BrainHeart Infusion and (c) Nutrient Broth. The plots represent the number of Log10 CFU/ml from resuspended feces. (d) Plot of the body weight (g) of the animals during the 6 days of antibiotic treatment.

\section{Abbreviations}

CFU: colony forming unit; TMB: 3,3',5,5'-tetramethylbenzidine; LB: Luria-Bertani; slgA: secretory immunoglobulin A; ABX: antibiotic.

\section{Authors' contributions}

$M A, C A$, and $C E$ conceived and designed the experiments. $C V, M H, C A$, and $C E$ performed the experiments. CV, MH, MA, CA, and CE analyzed the data. CV, $\mathrm{MH}, \mathrm{MA}, \mathrm{CA}$, and CE contributed reagents, materials, and analysis tools. CA and CE wrote the paper. All authors read and approved the final manuscript.

\section{Author details}

${ }^{1}$ Institute of Virology, University of Zurich, Winterthurerstrasse 266a, 8057 Zurich, Switzerland. ${ }^{2}$ Laboratory for Animal Model Pathology, Institute of Pathology, Vetsuisse, University of Zurich, Zurich, Switzerland. ${ }^{3}$ rqmicro AG, Schlieren, Switzerland.

\section{Acknowledgements}

We thank Bogdana Salathé-Gorza and Lettebrhan Ghebre-Tecle for excellent technical support at the animal facility. We would like to thank Sabine Wunderlin for her excellent technical assistance.

\section{Competing interests}

The authors declare that they have no competing interests.

\section{Availability of data and materials}

All data generated or analyzed during this study are included in this published article and its Additional files. 


\section{Consent for publication \\ Not applicable.}

\section{Ethics approval and consent to participate}

All the mouse experiments were performed according to the guidelines of the animal experimentation law (SR 455.163; TV) of the Swiss federal government. The Cantonal Veterinary Office of Zurich, Switzerland, approved the protocols under animal experimentation number 104/2012.

\section{Funding}

This research was funded by a PARAVAC European Commission grant (reference number 265862/FP-7-KBBE).

\section{Publisher's Note}

Springer Nature remains neutral with regard to jurisdictional claims in published maps and institutional affiliations.

Received: 13 August 2018 Accepted: 16 November 2018 Published online: 26 November 2018

\section{References}

1. Duc L, Hong HA, Fairweather N, Ricca E, Cutting SM. Bacterial spores as vaccine vehicles. Infect Immun. 2003;71:2810-8.

2. Ricca E, Baccigalupi L, Cangiano G, De Felice M, Isticato R. Mucosal vaccine delivery by non-recombinant spores of Bacillus subtilis. Microb Cell Fact. 2014;13:115.

3. Rosales-Mendoza S, Angulo C. Bacillus subtilis comes of age as a vaccine production host and delivery vehicle. Expert Rev Vaccines. 2015; 14:1135-48

4. Huang K, Chen C, Shen Q, Rosen BP, Zhao FJ. Genetically engineering Bacillus subtilis with a heat-resistant arsenite methyltransferase for bioremediation of arsenic-contaminated organic waste. Appl Environ Microbiol. 2015;81:6718-24.

5. Samuel J, Paul ML, Ravishankar H, Mathur A, Saha DP, Natarajan C, Mukherjee $A$. The differential stress response of adapted chromite mine isolates Bacillus subtilis and Escherichia coli and its impact on bioremediation potential. Biodegradation. 2013;24:829-42.

6. Gonzalez-Pastor JE. Cannibalism: a social behavior in sporulating Bacillus subtilis. FEMS Microbiol Rev. 2011;35:415-24.

7. Higgins D, Dworkin J. Recent progress in Bacillus subtilis sporulation. FEMS Microbiol Rev. 2012;36:131-48.

8. Copland A, Diogo GR, Hart P, Harris S, Tran AC, Paul MJ, Singh M, Cutting SM, Reljic R. Mucosal delivery of fusion proteins with Bacillus subtilis spores enhances protection against tuberculosis by Bacillus Calmette-Guerin. Front Immunol. 2018;9:346

9. Hong HA, Hitri K, Hosseini S, Kotowicz N, Bryan D, Mawas F, Wilkinson AJ, van Broekhoven A, Kearsey J, Cutting SM. Mucosal antibodies to the C terminus of toxin A prevent colonization of Clostridium difficile. Infect Immun. 2017. https://doi.org/10.1128/IAl.01060-16.

10. Huang JM, Hong HA, Van Tong H, Hoang TH, Brisson A, Cutting SM. Mucosal delivery of antigens using adsorption to bacterial spores. Vaccine. 2010;28:1021-30.

11. Potocki W, Negri A, Peszynska-Sularz G, Hinc K, Obuchowski M, Iwanick A. The combination of recombinant and non-recombinant Bacillus subtilis spore display technology for presentation of antigen and adjuvant on single spore. Microb Cell Fact. 2017;16:151.

12. Reljic R, Sibley L, Huang JM, Pepponi I, Hoppe A, Hong HA, Cutting SM. Mucosal vaccination against tuberculosis using inert bioparticles. Infect Immun. 2013;81:4071-80.

13. Vlamakis $H$, Chai $Y$, Beauregard $P$, Losick R, Kolter R. Sticking together: building a biofilm the Bacillus subtilis way. Nat Rev Microbiol. 2013:11:157-68.

14. Hong HA, Khaneja R, Tam NM, Cazzato A, Tan S, Urdaci M, Brisson A, Gasbarrini A, Barnes I, Cutting SM. Bacillus subtilis isolated from the human gastrointestinal tract. Res Microbiol. 2009;160:134-43.

15. Tam NK, Uyen NQ, Hong HA, le Duc H, Hoa TT, Serra CR, Henriques AO, Cutting SM. The intestinal life cycle of Bacillus subtilis and close relatives. J Bacteriol. 2006;188:2692-700.
16. Branda SS, Gonzalez-Pastor JE Ben-Yehuda S, Losick R, Kolter R. Fruiting body formation by Bacillus subtilis. Proc Natl Acad Sci U S A. 2001:98:11621-6.

17. Vlamakis H, Aguilar C, Losick R, Kolter R. Control of cell fate by the formation of an architecturally complex bacterial community. Genes Dev. 2008;22:945-53.

18. Vogt CM, Schraner EM, Aguilar C, Eichwald C. Heterologous expression of antigenic peptides in Bacillus subtilis biofilms. Microb Cell Fact. 2016;15:137.

19. Veening JW, Smits WK, Hamoen LW, Kuipers OP. Single cell analysis of gene expression patterns of competence development and initiation of sporulation in Bacillus subtilis grown on chemically defined media. J Appl Microbiol. 2006;101:531-41.

20. Larsen N, Thorsen L, Kpikpi EN, Stuer-Lauridsen B, Cantor MD, Nielsen B, Brockmann E, Derkx PM, Jespersen L. Characterization of Bacillus spp. strains for use as probiotic additives in pig feed. Appl Microbiol Biotechnol. 2014;98:1105-18

21. Tompkins TA, Xu X, Ahmarani J. A comprehensive review of post-market clinical studies performed in adults with an Asian probiotic formulation. Benef Microbes. 2010;1:93-106.

22. Zokaeifar H, Babaei N, Saad CR, Kamarudin MS, Sijam K, Balcazar JL. Administration of Bacillus subtilis strains in the rearing water enhances the water quality, growth performance, immune response, and resistance against Vibrio harveyi infection in juvenile white shrimp, Litopenaeus vannamei. Fish Shellfish Immunol. 2014:36:68-74.

23. Vogt CM, Armua-Fernandez MT, Tobler K, Hilbe M, Aguilar C, Ackermann M, Deplazes P, Eichwald C. Oral application of recombinant Bacillus subtilis spores to dogs results in a humoral response against specific Echinococcus granulosus paramyosin and tropomyosin antigens. Infect Immun. 2018. https://doi.org/10.1128/IAl.00495-17.

24. Stubbendieck RM, Straight PD. Multifaceted interfaces of bacterial competition. J Bacteriol. 2016;198:2145-55

25. Lloyd DP, Allen RJ. Competition for space during bacterial colonization of a surface. J R Soc Interface. 2015;12:0608.

26. Shan M, Gentile M, Yeiser JR, Walland AC, Bornstein VU, Chen K, He $B$, Cassis L, Bigas A, Cols M, et al. Mucus enhances gut homeostasis and oral tolerance by delivering immunoregulatory signals. Science. 2013:342:447-53.

27. Fraize M, Sarciron ME, Azzouz S, Issaadi N, Bosquet G, Petavy AF. Immunogenicity of two Echinococcus granulosus antigens EgA31 and EgTrp in mice. Parasitol Res. 2005;96:113-20.

28. Petavy AF, Hormaeche $\mathrm{C}$, Lahmar S, Ouhelli H, Chabalgoity A, Marchal T, Azzouz S, Schreiber F, Alvite G, Sarciron ME, et al. An oral recombinant vaccine in dogs against Echinococcus granulosus, the causative agent of human hydatid disease: a pilot study. PLoS Negl Trop Dis. 2008;2:e125

29. Budke CM, Deplazes P, Torgerson PR. Global socioeconomic impact of cystic echinococcosis. Emerg Infect Dis. 2006;12:296-303.

30. Deplazes P, Rinaldi L, Alvarez Rojas CA, Torgerson PR, Harandi MF, Romig T, Antolova D, Schurer JM, Lahmar S, Cringoli G, et al. Global distribution of alveolar and cystic echinococcosis. Adv Parasitol. 2017;95:315-493.

31. Organization WH. Accelerating work to overcome the global impact of neglected tropical diseases. A roadmap for implementation. Geneva: WHO Press; 2012.

32. Ciabattini A, Parigi R, Isticato R, Oggioni MR, Pozzi G. Oral priming of mice by recombinant spores of Bacillus subtilis. Vaccine. 2004:22:4139-43.

33. Lega T, Weiher P, Obuchowski M, Nidzworski D. Presenting influenza A M2e antigen on recombinant spores of Bacillus subtilis. PLOS ONE. 2016:11:e0167225

34. Li L, Hu X, Wu Z, Xiong S, Zhou Z, Wang X, Xu J, Lu F, Yu X. Immunogenicity of self-adjuvanticity oral vaccine candidate based on use of Bacillus subtilis spore displaying Schistosoma japonicum 26 kDa GST protein. Parasitol Res. 2009:105:1643-51.

35. Permpoonpattana P, Hong HA, Phetcharaburanin J, Huang JM, Cook J, Fairweather NF, Cutting SM. Immunization with Bacillus spores expressing toxin A peptide repeats protects against infection with Clostridium difficile strains producing toxins A and B. Infect Immun. 2011:79:2295-302.

36. Stasilojc M, Hinc K, Peszynska-Sularz G, Obuchowski M, Iwanicki A. Recombinant Bacillus subtilis spores elicit Th1/Th17-polarized immune response in a murine model of Helicobacter pylori vaccination. Mol Biotechnol. 2015;57:685-91. 
37. Sun $H$, Lin Z, Zhao L, Chen T, Shang M, Jiang H, Tang Z, Zhou X, Shi M, Zhou L, et al. Bacillus subtilis spore with surface display of paramyosin from Clonorchis sinensis potentializes a promising oral vaccine candidate. Parasit Vectors. 2018;1 1:156.

38. Tang Z, Shang M, Chen T, Ren P, Sun H, Qu H, Lin Z, Zhou L, Yu J, Jiang $H$, et al. The immunological characteristics and probiotic function of recombinant Bacillus subtilis spore expressing Clonorchis sinensis cysteine protease. Parasit Vectors. 2016;9:648.

39. Zhou Z, Gong S, Li XM, Yang Y, Guan R, Zhou S, Yao S, Xie Y, Ou Z, Zhao J, Liu Z. Expression of Helicobacter pylori urease B on the surface of Bacillus subtilis spores. J Med Microbiol. 2015;64:104-10.

40. Croswell A, Amir E, Teggatz P, Barman M, Salzman NH. Prolonged impact of antibiotics on intestinal microbial ecology and susceptibility to enteric Salmonella infection. Infect Immun. 2009;77:2741-53.
41. Guo X, Chen DD, Peng KS, Cui ZW, Zhang XJ, Li S, Zhang YA. Identification and characterization of Bacillus subtilis from grass carp (Ctenopharynodon idellus) for use as probiotic additives in aquatic feed. Fish Shellfish Immunol. 2016;52:74-84.

42. Cohn M. Dissecting the two models of TCR structure-function relationships. Immunol Res. 2016;64:795-803.

43. Nicolas L, Cols M, Choi JE, Chaudhuri J, Vuong B. Generating and repairing genetically programmed DNA breaks during immunoglobulin class switch recombination. F1000Res. 2018:7:458
Ready to submit your research? Choose BMC and benefit from:

- fast, convenient online submission

- thorough peer review by experienced researchers in your field

- rapid publication on acceptance

- support for research data, including large and complex data types

- gold Open Access which fosters wider collaboration and increased citations

- maximum visibility for your research: over $100 \mathrm{M}$ website views per year

At BMC, research is always in progress.

Learn more biomedcentral.com/submissions 